Gut and Liver, Vol. 13, No. 1, January 2019, pp. 104-113

\title{
Incidence and Overall Survival of Biliary Tract Cancers in South Korea from 2006 to 2015: Using the National Health Information Database
}

\author{
Byung-Woo Kim ${ }^{1}$, Chang-Mo Oh ${ }^{2}$, Hwa Young Choi ${ }^{1}$, Joong-Won Park ${ }^{1,3}$, Hyunsoon $\mathrm{Cho}^{1}$, and Moran $\mathrm{Ki}^{1}$ \\ ${ }^{1}$ Department of Cancer Control and Population Health, Graduate School of Cancer Science and Policy, National Cancer Center, Goyang, \\ ${ }^{2}$ Department of Preventive Medicine, Kyung Hee University School of Medicine, Seoul, and ${ }^{3}$ Center for Liver Cancer, National Cancer Center, \\ Goyang, Korea
}

Background/Aims: There have been no nationwide studies to investigate the trends in incidence and 5-year survival rates of intra- and extrahepatic bile duct cancers and gallbladder cancer. Therefore, our study aimed to describe the incidence and 5-year survival rates of biliary tract cancers by subsites in South Korea. Methods: A total of 86,134 patients with biliary tract cancers were selected from the National Health Information Database. Age-standardized incidence rates and annual percentage changes were calculated. Lifetable methods and log-rank tests were used to determine the differences in survival rates. Cox-proportional hazard models were used to estimate the hazard ratio of the patients with biliary tract cancers. Results: The incidence rate of intrahepatic bile duct cancer decreased by $1.3 \%$ annually from 8.8 per 100,000 in 2006 to 7.8 per 100,000 in 2015. Extrahepatic bile duct cancer also showed a decreasing trend by $2.2 \%$ per year from 8.7 per 100,000 in 2006 to 6.7 per 100,000 in 2015. Gallbladder cancer showed the greatest decline, with an annual percentage change of $2.8 \%$ from 6.3 per 100,000 to 5.2 per 100,000 during the same period. The 5-year survival rates were $30.0 \%$ in gallbladder cancer, $27.8 \%$ in extrahepatic bile duct cancer, and $15.9 \%$ in intrahepatic bile duct cancer. Conclusions: The overall incidence rates of intrahepatic and extrahepatic bile duct cancer and gallbladder cancer decreased from 2006 to 2015. Among biliary tract cancers, intrahepatic bile duct cancers exhibited the highest incidence rate and the worst survival rate. (Gut Liver 2019;13:104-113)

Key Words: Incidence; Survival; Gallbladder neoplasm; Cholangiocarcinoma

\section{INTRODUCTION}

Biliary tract cancers include cancers that occur in the intraand extrahepatic bile ducts as well as the gallbladder. ${ }^{1}$ According to national cancer statistics in 2014 year reported by Korea Central Cancer Registry (KCCR), there were 5,576 patients with gallbladder and other biliary tract cancers (ICD-10: C23-C24) in South Korea. Ranked 9th in its number of incidence, these cancers are rather common in the Korean population compared to western countries. ${ }^{2}$ However, the cancer statistics published by KCCR did not distinguish between gallbladder cancers and biliary tract cancers, and intrahepatic bile duct cancers are included as the part of liver cancer. These drawbacks hamper interpretation of these results, and the applications in the clinical setting.

Recently, some epidemiological studies in the western countries have shown increasing incidences of intrahepatic bile duct cancers in the majority of European countries (excluding Denmark) and the United States, ${ }^{3-7}$ while those of extrahepatic bile duct cancers have decreased in the United States and European countries. ${ }^{4,5,7}$ In addition, the incidence rates of gallbladder cancers have been steadily decreasing over the past 30 years in both male and female patients in the United States and United Kingdom. ${ }^{9,10}$

Incidence rate of intrahepatic and extrahepatic bile duct cancers in an Asian population is extremely high compared to the Caucasian population from the United States. ${ }^{2,10,11}$ The prevalence of intrahepatic and extrahepatic bile duct cancers was also high in Korea, but a reduction in the prevalence of both intrahepatic and extrahepatic bile duct cancers is expected due to recent decreases in the prevalence of hepatitis B and clonorchiasis, which were major risk factors for intrahepatic and extrahepatic

\footnotetext{
Correspondence to: Moran Ki

Department of Cancer Control and Population Health, Graduate School of Cancer Science and Policy, National Cancer Center, 323 Ilsan-ro, Ilsandong-gu, Goyang 10408, Korea

Tel: +82-31-920-2736, Fax: +82-2-6455-8699, E-mail: moranki@ncc.re.kr

Received on February 26, 2018. Revised on April 8, 2018. Accepted on April 25, 2018. Published online September 21,2018

pISSN 1976-2283 eISSN 2005-1212 https://doi.org/10.5009/gnl18105

Byung-Woo Kim and Chang-Mo Oh contributed equally to this work as first authors.

@ This is an Open Access article distributed under the terms of the Creative Commons Attribution Non-Commercial License (http://creativecommons.org/licenses/by-nc/4.0) which permits unrestricted non-commercial use, distribution, and reproduction in any medium, provided the original work is properly cited.
} 
bile duct cancers. ${ }^{12,13}$ However, there are rare previous studies in the Korean population that assess how the prevalence of intra-, and extrahepatic bile duct cancers and gallbladder cancers is changing.

According to the data from KCCR, 5-year survival rate of patients with gallbladder, and other biliary duct cancers from 2010 to 2014 was $29.2 \%$, which has been steadily increasing from the 5-year survival rate of $17.3 \%$ in the patients between 1993 and $1995 .^{2}$ Nevertheless, intrahepatic and extrahepatic bile duct cancers, and gallbladder cancers have clinically distinct genetic characteristics and prognostic factors. ${ }^{14,15}$ Even within extrahepatic biliary tract cancers, the 5-year survival rate differed within a cohort of Japanese patients based on the location of the lesion (24.2\%, 39.1\%, and 61.3\% for perihilar, distal, and ampullary tumors, respectively). ${ }^{16}$ The 5-year survival rate for patients with gallbladder cancer was 38.9\%, indicating different prognosis based on the anatomical location of the cancerous lesion. ${ }^{16}$ For clinicians and patients with biliary tract cancer, the 5-year survival rate for each cancer type provides crucial information and evidence for deciding on a treatment, and estimating prognosis. However, there are no representative datasets of Korean populations, regarding the 5-year survival rates of intrahepatic biliary tract cancers, extrahepatic biliary tract cancers, and gallbladder cancers based on the specific location of the lesion.

Therefore, in this study, we performed a comprehensive descriptive study of the trends in incidence rates of intrahepatic and extrahepatic bile duct cancers, and gallbladder cancers, as well as the survival rate in Korean patients with biliary tract cancer, based on the specific location of the lesion, using National Health Information Database (NHID) of the National Health Insurance Service (NHIS), which represents the entire Korean population.

\section{MATERIALS AND METHODS}

\section{Data source}

The health insurance coverage rate in Korea is over 98\%, indicating that the database can be used to represent the medical service usage of the entire Korean population. Furthermore, the NHIS provides customized data for research purposes after removing personal information from the database. Information on demographic variables such as sex, age at diagnosis, region of residence and medical records such as diagnosis, the medical institution, date of diagnosis, prescription, and medical expenses are included in the NHID. In addition, an updated date of death for patients was also provided, utilizing an up-to-date database from the Ministry of Public Administration and Security. ${ }^{17,18}$ Moreover, the severe cancer registration program in Korea from September 2005 allows financial support for the patients, and the information on patients registered for this specialized program allows improved accuracy of diagnosis for these cancer patients. Lastly, we obtained data on the incidence rates of biliary tract cancers according to specific locations between 1999, and 2014 from the Korean National Cancer Incidence Database (from KCCR), in order to compare with the incidence rate from NHIS database. This study was approved by National Cancer Center institutional review board (IRB; NCC2017-0034) and did not require patient consent as the study was retrospective, using secondary data which did not contain any personal information.

\section{Study population}

Using the NHID, we assessed the incidence-, and the survival rates of patients with biliary tract cancer, based on the anatomical sites of the cancer origin. Data on the patients diagnosed with intrahepatic bile duct cancers (ICD10: C22.1), extrahepatic bile duct cancers (ICD10: C24), and gallbladder cancers (ICD10: C23) were collected from NHID between 2002 and 2015. ${ }^{6}$ The following method was used to estimate the data of the patients with biliary tract cancer based on the location of the lesion: First, using the data from the initial date of diagnosis, patients who did not have a medical history of cancer diagnosis on the same location were defined as new cancer patients. The initial date of diagnosis was defined based on the main disease code. In cases with a previous history of an identical diagnosis of a sub-disease within 60 days of the prior diagnosis, the date of diagnosis of the sub-disease was used as the initial date of diagnosis; second, in order to improve diagnostic accuracy, we excluded patients who were not registered to the financial aid program for cancer patients (code: V193, V027). Therefore, data from 2002 to 2005 were excluded from the analysis, as the financial aid program for cancer patients was not available during this period. Furthermore, patients who were younger than 20 years of age at the initial date of diagnosis were excluded from the study. Because, there were only nine patients newly diagnosed as gallbladder, and other biliary tract cancers from 1999 to 2014 according to the KCCR. ${ }^{19}$ In addition, epidemiological or biological characteristics of cancer among children and adolescents was different from those of adults. ${ }^{20,21}$ Patients with multiple cancers were only included based on the first cancer diagnosis, per the initial date of diagnosis. In cases where the initial date of diagnosis was identical, admission records were taken over outpatient clinic visits. If this information was identical, the diagnosis with higher medical expense was chosen.

We assessed the survival and the date of death in the finalized cohort of 86,134 patients with biliary tract cancer $(34,695$ with intrahepatic bile duct cancer; 29,063 with extrahepatic bile duct cancer; and 22,376 with gallbladder cancer) that were included in the study (2006 to 2015). The region of incidence was categorized into 16 different cities and provinces, based on the location of the medical institution at the initial diagnosis. The Sejong city was considered part of Chungnam province. Of the 381 patients who did not have location information at the 
initial diagnosis, the information of region for 106 patients were corrected using the data from consultations that occurred in the same year. The remaining 275 cancer patients were categorized into "unknown" for the region.

For the comorbidities in the patients with biliary tract cancer, the date of diagnosis with the disease $\geq 1$ time as main disease or $\geq 3$ times as sub-disease was used as a standard until the date of biliary tract cancer diagnosis. From the entire dataset between 2002 and 2015, the following diseases were considered: Clonorchis sinensis infection (ICD10: B66 excluding B66.4); hepatitis B (ICD10: B16, B17.0, B18.0, B18.1); hepatitis C (ICD10: B17.1, B18.2); alcoholic liver disease (ICD10: K70); type 2 diabetes (ICD10: E11); nonalcoholic liver disease (ICD10: K75.8, K76.0); gallstones (ICD10: K80); and other biliary tract diseases (ICD10: K83). Diagnostic data on the exclusionary diseases were excluded from the calculation of standard.

Patients with gallbladder cancer in Korea included the patients registered for the financial aid program (code: V193, V027) with the main diseases of intrahepatic bile duct cancer (ICD10: C22.1), extrahepatic bile duct cancer (ICD10: C24), or gallbladder cancer (ICD10: C23), from the NHID. Patients under 20 years of age were not included.

\section{Statistical analysis}

Independent t-test was performed to compare the mean age between men and women and chi-square test was used to compare the frequency of comorbid diseases between men and women by sub-sites of biliary tract cancer. The Korean mid-year population obtained from Statistics Korea was used to calculate the incidence rate (i.e., the denominator). ${ }^{22}$ Using Korean midyear population in 2010 as standard population, the age and sex standardized incidence rate per 100,000 people was calculated after adjusting for sex and age. The incidence rate using the data from the KCCR was calculated using the same method. After logarithmic transformation of the incidence rate, a linear regression model was used to calculate the annual percentage change (APC). The same denominator population was used in the calculation of prevalence, and the prevalence per 100,000 people was calculated after adjusting for sex and age. The survival rate of patients with biliary tract cancer was calculated using the life table method, and the survival rate for each duration (1 to 10 years from the date of cancer diagnosis) was calculated. For age- groups, patients were divided into two groups $(\geq 70$ years, and 20 to 69 years), and a log-rank test was used to assess the difference in the survival rates between the two. Lastly, the Cox proportional hazard model was used to estimate risk of mortality by age, sex and the year of diagnosis.

The p-values less than 0.05 were considered significant. SAS version 9.4 (SAS Institute, Cary, NC, USA) was used to perform statistical analysis and R software version 3.4.1 (Vienna, Austria, https://www.r-project.org/) was used for statistical analysis and graphical presentation.

\section{RESULTS}

\section{Study population}

From the NHID between 2002 and 2015, data on 171,350 patients were collected, comprising 160,914 patients who do not have medical history of biliary tract cancer as of the initial date of diagnosis, or biliary tract cancer is a main disease; and 10,436 patients who had medical history of biliary tract cancer as a sub-disease, but not a main disease code and the duration of cancer diagnosis was within 60 days. The following data were excluded from the analysis: 45,610 patients who were diagnosed with biliary tract cancer from 2002 to 2005; 16,490 patients who had no information on the financial aid program for cancer patients; and 15 patients who were younger than 20 years old. After appropriate management of 23,101 patients with multiple cancers $(7,983$ patients with intrahepatic bile duct cancer; 11,654 with extrahepatic bile duct cancer; and 3,464 with gallbladder cancer), there were 86,134 patients included in the finalized cohort with a diagnosis between 2006 and 2015. Among these patients, 34,695 patients (40.3\%) had intrahepatic bile duct cancer, 29,063 patients (33.7\%) had extrahepatic bile duct cancer, and 22,376 patients (26.0\%) had gallbladder cancer (Fig. 1).

Based on sex, both intrahepatic and extrahepatic bile duct cancers were more prevalent in men (male 62.1\% vs female $37.9 \%$, male $57.5 \%$ vs female $42.5 \%$, respectively). However, gallbladder cancers were more prevalent in female patients (55.4\%) compared to male patients (44.6\%). Mean age at diagnosis was higher in female patients than that in male patients for all three cancer types (male: 66.4 years old vs female: 70.2 years old for intrahepatic bile duct cancers $[\mathrm{p}<0.001]$, male: 67.4 years old vs female: 70.8 years old for extrahepatic bile duct cancers $[\mathrm{p}<0.001]$ and male: 67.7 years old vs female: 69.9 years old for gallbladder cancers $[p<0.001])$ (Table 1).

\section{Comorbidities of biliary tract cancer patients}

Type 2 diabetes was the most prevalent comorbidity $(\geq 30 \%)$ in patients with biliary tract cancer for all three cancer types (Table 1). Patients with intra- and extrahepatic bile duct cancer exhibited more than two times higher in frequency of other biliary tract diseases, compared to patients with gallbladder cancer, while gallstones were more prevalent in gallbladder cancer patients. Alcoholic liver disease was 5-times more prevalent in male patients than that in female patients. $C$. sinensis infection was the least prevalent comorbidity in all three cancer types, with $<2 \%$ in male and $<1 \%$ in female patients.

\section{Incidence and prevalence}

Incidence rates of biliary tract cancers have decreased for all three cancer types from 2006 to 2015 . While the incidence rates in 2006 were 8.8, 8.7, and 6.3 per 100,000 people for intrahepatic bile duct cancer, extrahepatic bile duct cancer, and 


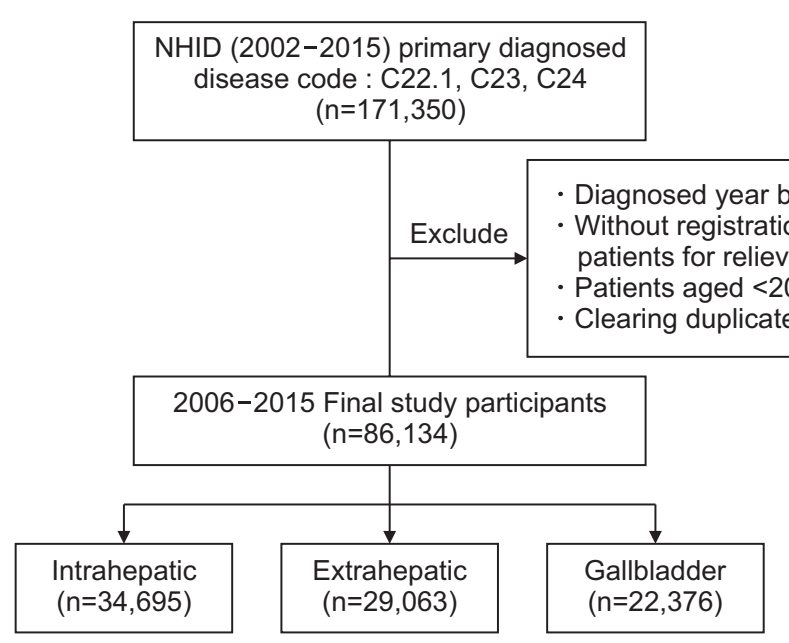

gallbladder cancer, respectively, the corresponding rates in 2015 were 7.8 , 6.7, and 5.2 per 100,000 people, respectively. Moreover, from the analysis of APC, the incidence rates have been steadily decreasing with APC values of $-1.3 \%$ for intrahepatic bile duct cancers, $-2.2 \%$ for extrahepatic bile duct cancers, and $-2.8 \%$ for gallbladder cancers, and these differences were statistically significant (Table 2). The incidence rate of intrahepatic bile duct cancers in female patients remained stable, unlike what was observed in the male patients (Fig. 2).

When comparing the incidence rates of biliary tract cancers with those of KCCR based on anatomical locations, the incidence rate of intrahepatic bile duct cancers was slightly higher in the data from KCCR. The differences in the incidence rates of extrahepatic bile duct cancers and gallbladder cancers became smaller when more recent data was considered (Table 2, Supplementary Fig. 1).

From the data on incidence rate per region between 2006 and 2015, the region with highest incidence rate of intrahepatic bile duct cancer was Gyeongnam province, with 14.6 per 100,000 people, and the region with lowest incidence rate was the Incheon city, with 6.5 per 100,000 people (ratio: 2.5-fold). For extrahepatic bile duct cancers, the region with highest incidence rate was Gyeongnam province, with 9.6 per 100,000 people, while the Daejeon city had lowest incidence rate, with 5.9 per 100,000 people (ratio: 1.6-fold). Lastly, the region with the highest incidence rate of gallbladder cancers was the Ulsan city, with 7.5 per 100,000 people, and the region with lowest incidence rate was the Incheon city, with 4.7 per 100,000 people (ratio: 1.6) (Fig. 3).

Using the most recent data (2015) as a standard, we assessed the incidence rates of biliary tract cancers based on age. Intrahepatic bile duct cancers were most prevalent in all age groups, followed by extrahepatic bile duct cancers, and gallbladder cancers. There were rarely any patients in the 20 to 30 years old group, and the incidence rate started to increase in the patients in their 40s with a peak rate in the patients in their 70s (Supple-
Fig. 1. Flowchart for selecting the study populations using the National Health Information Database (NHID) in South Korea. mentary Fig. 2). When we assessed the prevalence based on age, patients in their 40s started to exhibit a higher prevalence of extrahepatic bile duct cancers compared to intrahepatic bile duct cancers, with highest prevalence out of all biliary tract cancers (Supplementary Fig. 3).

\section{Survival}

Five-year survival in patients with biliary tract cancer was $30 \%$ for gallbladder cancer, followed by $27.8 \%$ for extrahepatic bile duct cancers, and 15.9\% for intrahepatic bile duct cancers. Intrahepatic bile duct cancers had the lowest survival rate compared to the other two cancer types. Similarly, for median survival, patients with intrahepatic bile duct cancer had the lowest median survival (8.0 months) compared to those with extrahepatic bile duct cancer (16.6 months), or gallbladder cancer (10.7 months) (Table 3). There was a decreasing trend in 5-year survival rates for intrahepatic bile duct cancer from 17.7\% in 2006 to 15.2 in 2011, whereas there were no clear trends in 5-year survival rates for patients with extrahepatic bile duct cancer and gallbladder cancer (Supplementary Table 1). Younger age group ( $<70$ years) has higher 5-year survival rates (Intrahepatic bile duct cancer: 21\%, Extrahepatic bile duct cancer: 39\%, gallbladder cancer: 40\%) compared to older age group ( $\geq 70$ years) (intrahepatic bile duct cancer, 10\%; extrahepatic bile duct cancer, 18\%; gallbladder cancer, 21\%) in all three tumor groups ( $<<0.001$ ) (Supplementary Figs 4 and 5).

We then analyzed the risk of mortality in patients with biliary tract cancer, after adjusting for the year of diagnosis, sex, and age (Supplementary Table 2). As the year of diagnosis was more recent, the risk of mortality decreased from 2006 to 2015 in all three tumor groups (intrahepatic bile duct cancer: hazards ratio $[H R]=0.996, p=0.041$; extrahepatic bile duct cancer: $H R=0.975$, $\mathrm{p}<0.001$; and gallbladder cancer: $H R=0.984, p<0.001)$. The risks of mortality for men compared to women in each cancer were as follows: intrahepatic bile duct $(\mathrm{HR}=1.038, \mathrm{p}=0.002)$; extrahepatic bile duct cancer $(H R=1.120, p<0.001)$; and gallbladder 


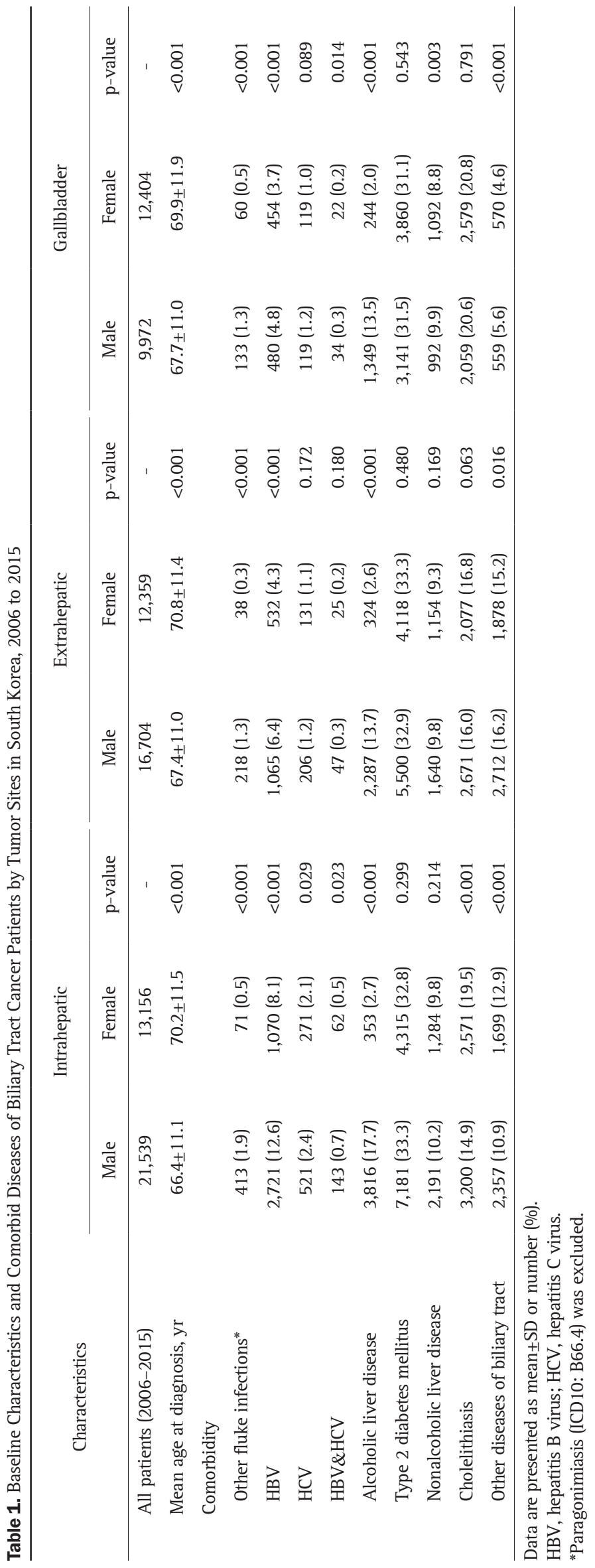


Table 2. Sex- and Age-Standardized Incidence Rates per 100,000 for Patients with Biliary Tract Cancers Aged $\geq 20$ Years in South Korea

\begin{tabular}{|c|c|c|c|c|c|c|}
\hline \multirow{2}{*}{ Data source (year) } & \multicolumn{2}{|c|}{ Intrahepatic } & \multicolumn{2}{|c|}{ Extrahepatic } & \multicolumn{2}{|c|}{ Gallbladder } \\
\hline & KNCID* & $\mathrm{NHID}^{\dagger}$ & $\mathrm{KNCID}^{*}$ & $\mathrm{NHID}^{\dagger}$ & KNCID* & $\mathrm{NHID}^{\dagger}$ \\
\hline 1999 & 4.5 & - & 7.1 & - & 5.5 & - \\
\hline 2000 & 4.1 & - & 6.8 & - & 5.7 & - \\
\hline 2001 & 4.4 & - & 7.3 & - & 6.0 & - \\
\hline 2002 & 5.2 & - & 6.9 & - & 6.3 & - \\
\hline 2003 & 5.6 & - & 7.1 & - & 6.0 & - \\
\hline 2004 & 6.4 & - & 7.6 & - & 5.9 & - \\
\hline 2005 & 7.2 & - & 7.7 & - & 6.2 & - \\
\hline 2006 & 7.3 & 8.8 & 6.9 & 8.7 & 6.0 & 6.3 \\
\hline 2007 & 8.3 & 9.1 & 6.9 & 8.1 & 6.0 & 6.3 \\
\hline 2008 & 8.3 & 9.3 & 6.9 & 7.7 & 5.8 & 5.9 \\
\hline 2009 & 8.4 & 9.0 & 7.4 & 8.1 & 5.9 & 5.9 \\
\hline 2010 & 7.9 & 9.1 & 7.2 & 7.5 & 5.8 & 5.9 \\
\hline 2011 & 8.4 & 9.3 & 7.1 & 7.4 & 5.5 & 5.7 \\
\hline 2012 & 8.0 & 8.8 & 6.8 & 6.7 & 5.6 & 5.5 \\
\hline 2013 & 7.9 & 8.8 & 6.8 & 6.5 & 5.3 & 5.3 \\
\hline 2014 & 7.5 & 8.2 & 6.8 & 7.0 & 5.2 & 5.3 \\
\hline 2015 & - & 7.8 & - & 6.7 & - & 5.2 \\
\hline $\mathrm{APC}^{\ddagger}$ & - & -1.3 & - & -2.2 & - & -2.8 \\
\hline
\end{tabular}

Sex- and age-standardized incidence rates per 100,000 people were calculated. The mid-year population in 2010 was used as the standard population.

APC, annual percentage change.

*The Korea National Cancer Incidence Database (1999-2014); ${ }^{\dagger}$ The National Health Information Database in South Korea (2006-2015); ${ }^{\ddagger}$ APCs were statistically significant $(\mathrm{p}<0.05)$.
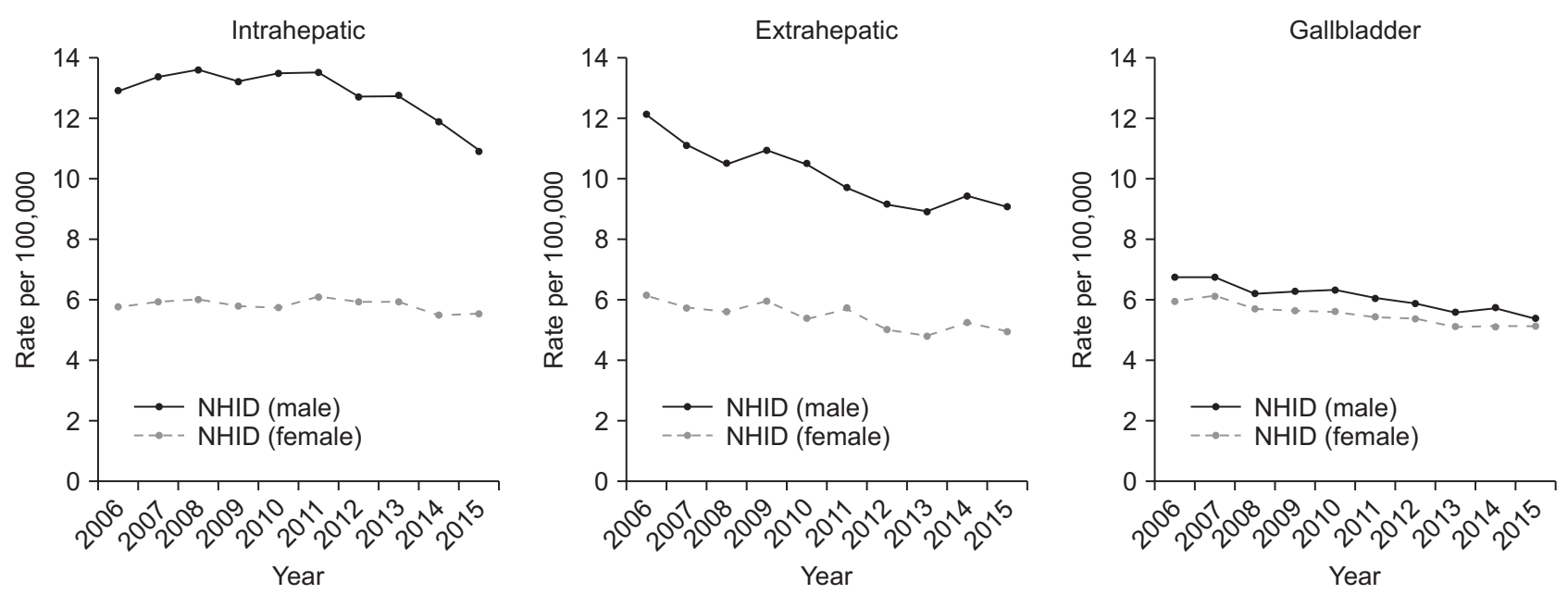

Fig. 2. Trends in age-standardized incidence rates by sex for biliary tract cancers in patients aged $\geq 20$ years using the National Health Information Database (NHID) in South Korea from 2006 to 2015. Age-standardized incidence rate per 100,000 people was calculated. The mid-year population in 2010 was used as the standard population.

cancer $(H R=1.050, p=0.003)$. The risks of mortality were higher in male patients in all three cancers even after adjusting for age and year of diagnosis. The risks of mortality based on age increased as the patient was older. More specifically, patients who were $\geq 80$ years of age exhibited the highest risk of mortal- ity for all cancer types compared to the patients in their 40s: intrahepatic bile duct cancer $(\mathrm{HR}=2.275, \mathrm{p}<0.001)$; extrahepatic bile duct cancer $(\mathrm{HR}=3.692, \mathrm{p}<0.001)$; and gallbladder cancer $(\mathrm{HR}=3.226, \mathrm{p}<0.001)$. 

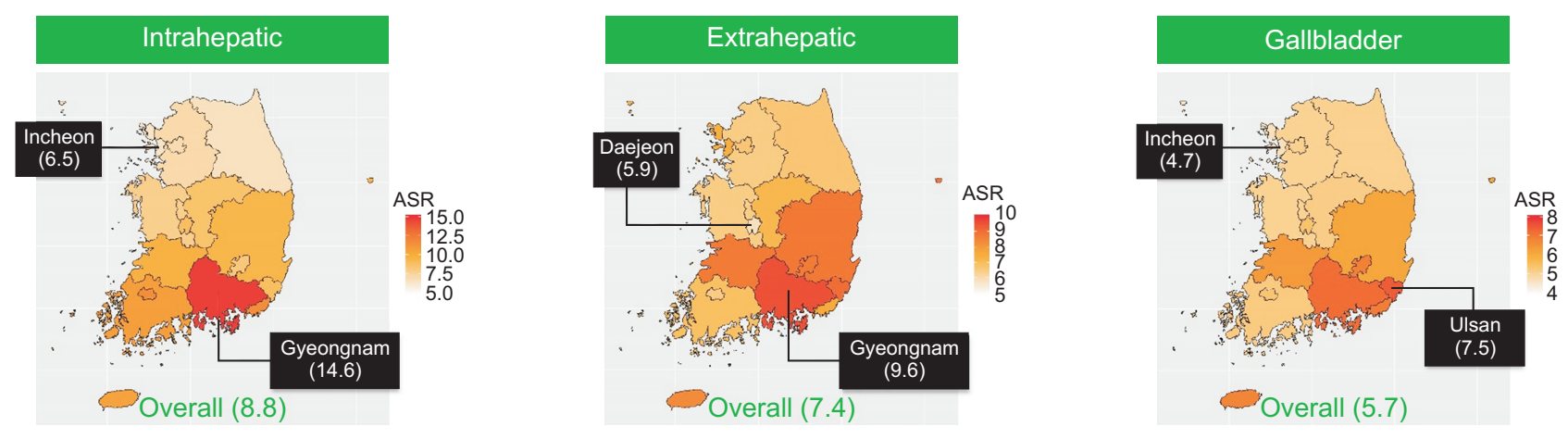

Fig. 3. Comparison of sex-, and age-standardized rates (ASR) for biliary tract cancers by region in South Korea from 2006 to 2015 . Sex- and agestandardized incidence rates per 100,000 people were calculated. The mid-year population in 2010 was used as the standard population.

Table 3. Overall Survival for Biliary Tract Cancers in South Korea, from 2006 to 2015

\begin{tabular}{|c|c|c|c|c|c|c|c|c|}
\hline \multirow{2}{*}{ Site } & \multirow{2}{*}{ Sex } & \multirow{2}{*}{ Number } & \multicolumn{5}{|c|}{ Observed survival rates, \% } & \multirow{2}{*}{ Survival, mo* } \\
\hline & & & $1-\mathrm{yr}$ & $2-\mathrm{yr}$ & $3-\mathrm{yr}$ & $4-\mathrm{yr}$ & $5-\mathrm{yr}$ & \\
\hline \multirow[t]{3}{*}{ Intrahepatic } & Both sexes & 34,695 & 39.8 & 25.7 & 20.1 & 17.5 & 15.9 & $8.0(2.8-23.3)$ \\
\hline & Male & 21,539 & 40.8 & 26.5 & 20.6 & 17.9 & 16.2 & $8.3(2.8-24.3)$ \\
\hline & Female & 13,156 & 38.3 & 24.2 & 19.2 & 16.8 & 15.4 & $7.5(2.7-21.8)$ \\
\hline \multirow[t]{3}{*}{ Extrahepatic } & Both sexes & 29,063 & 57.8 & 41.5 & 34.4 & 30.4 & 27.8 & $16.6(5.3-41.9)$ \\
\hline & Male & 16,704 & 58.6 & 42.0 & 34.3 & 30.2 & 27.3 & $17.1(5.5-41.9)$ \\
\hline & Female & 12,359 & 56.8 & 41.0 & 34.5 & 30.7 & 28.5 & $15.9(5.1-41.7)$ \\
\hline \multirow[t]{3}{*}{ Gallbladder } & Both sexes & 22,376 & 48.1 & 38.0 & 33.9 & 31.6 & 30.0 & $10.7(3.4-43.1)$ \\
\hline & Male & 9,972 & 49.0 & 39.0 & 34.6 & 32.0 & 30.4 & $11.4(3.4-44.5)$ \\
\hline & Female & 12,404 & 47.3 & 37.1 & 33.3 & 31.3 & 29.8 & $10.3(3.3-41.8)$ \\
\hline
\end{tabular}

The last follow-up date was June 30, 2017.

*Median (interquartile range).

\section{DISCUSSION}

This study showed that the overall incidence rates of intraand extrahepatic bile duct cancers and gallbladder cancers in Korean population have steadily decreased from 2006 to 2015. However, the incidence rate of intrahepatic bile duct cancer was not significantly changed in female patients, and the majority of patients with biliary tract cancer (78.3\%) screened in this study were elderly aged more than 60 years. Moreover, the incidence rates of biliary tract cancers were different based on the region, with Gyeongnam province and nearby cities exhibiting higher incidences of all three cancer types (intra- and extrahepatic bile duct cancer, and gallbladder cancer). Overall 5-year survival rates remained poor, with $15.9 \%$ for intrahepatic bile duct cancer, 27.8\% for extrahepatic bile duct cancer, and 30.0\% for gallbladder cancer. More specifically, our results highlight intrahepatic bile duct cancers as they exhibit lowest survival rate and highest incidence rate among biliary tract cancers.

The age and sex standardized incidence rates in 2015 year were 7.8, 6.7, and 5.2 per 100,000 people for intrahepatic bile duct cancer, extrahepatic bile duct cancer, and gallbladder cancer, respectively. Intrahepatic bile duct cancers exhibited the highest incidence rate, and this result was similar to other studies reporting higher incidence rate of intrahepatic bile duct cancers in Austrian, American, and Danish populations. ${ }^{3,4,7}$ However, these studies from the United States and Denmark did not include gallbladder cancers. ${ }^{3,423}$ On the other hands, studies from Canada, Japan, and France have shown higher incidence rates of extrahepatic bile duct cancer, although the Japanese and French studies did not include gallbladder cancers. ${ }^{5,6,24}$ The incidence rates of both intra- and extrahepatic bile duct cancers have increased in Canada, but the incidence rate of intra-, and extrahepatic bile duct cancer increased and decreased respectively in Austria. In Japan, the changes in incidence rates were different based on sex, with increased incidence rates of intrahepatic bile duct cancer in male patients and decreased incidence rates of extrahepatic bile duct cancer in female patients. As shown in the above studies, the incidence rates of biliary tract cancers based on the location of the lesion were varied between the different countries. However, these studies reported 
only an increase in the incidence rate of intrahepatic bile duct cancer since 2010, and no decreased incidence rate of intrahepatic bile duct cancer was observed. On the other hands, in this study, we confirmed a reduced incidence rate of intrahepatic bile duct cancers since 2010 in a Korean population. This reduction is likely mainly due to a decreased prevalence of $C$. sinensis infection, and hepatitis $\mathrm{B}$, which are the key risk factors of biliary tract cancers. ${ }^{12,13}$ The incidence rates of gallbladder cancer shows decreasing trends in South Korea as also shown in the United States and United Kingdom. ${ }^{9,10}$ However, there was no exact explanation why gallbladder cancer has declined across the countries. For patients with gall stones or cholecystitis, the frequency of gallbladder resection has been increased. These widespread use of cholecystectomy may be partly attributed to decrease in gallbladder cancer incidence. ${ }^{25,26}$ However, the decrease in absolute risk for gallbladder cancer was very small and controversies remained. ${ }^{25}$

Although roughly $10 \%$ of biliary tract cancers in the Korean population are estimated to be caused by $C$. sinensis infection, ${ }^{12}$ only $1.3 \%$ to $1.9 \%$ of male patients and $0.3 \%$ to $0.5 \%$ of female patients included in this study had $C$. sinensis infection, which is relatively low. However, since most patients who were diagnosed with $C$. sinensis infection did not develop biliary tract cancers, and the proportion of patients who were diagnosed with both $C$. sinensis infection and biliary tract cancer was likely very low, we expect that there are a significant number of patients who were diagnosed with biliary tract cancer without knowledge of an infection by $C$. sinensis, however these patients could not be distinguished using the NHID data.

It was well-known that the prevalence of $C$. sinensis, which was major risk factor for intra- or extrahepatic bile duct carcinoma, is higher around the Nakdong River due to eating the raw-fish in this area. ${ }^{12,27}$ In addition, it was reported that the prevalence of hepatitis B and hepatitis C virus infection in Jeonnam and Gyeongnam regions was higher than other regions. ${ }^{28-32}$ However, it could not be explained why incidence rate of gallbladder cancer in Ulsan was the highest among regions in this study. Further research is necessary to investigate the cause of high incidence rate of gallbladder cancer in Ulsan region.

The 5-year survival rates of patients with biliary tract cancer (with data collected between 2006 and 2015) based on the location of the lesion were: $15.9 \%$ for intrahepatic bile duct cancer, 27.8\% for extrahepatic bile duct cancer, and 30.0\% for gallbladder cancer. Median survival times were 8.0 months for intrahepatic bile duct cancer, 10.7 months for gallbladder cancer, and 16.6 months for extrahepatic bile duct cancers, exhibiting poor prognosis in all three cancer types. Compared to the other international studies, the survival rate for patients with intrahepatic bile duct cancer was worse compared to patients in Canada, but better compared to patients in Austria. The survival rates for extrahepatic bile duct cancers, and gallbladder cancers were higher than in either of the two other countries. ${ }^{6,7}$
However, the survival rate for patients with gallbladder cancer was lower than that of Japan, and direct comparison could not be made for extrahepatic bile duct cancers as the analysis was performed based on specific location of the lesion. There was no information available for the survival rate for patients with intrahepatic bile duct cancer in Japan. ${ }^{16}$ Although we could not make comparisons with many other countries, all three studies from Korea, Canada, and Austria indicate that intrahepatic bile duct cancers exhibit the worst prognosis among cancers of the biliary tract. More specifically in the Korean population, intrahepatic bile duct cancers were the most prevalent cancer among the cancers of the biliary tract, yet exhibited the second most poor prognosis among all cancer types, after pancreatic cancers (ICD-10: C25). ${ }^{2}$ However, a direct comparison could not be made as the analysis method of survival rate was different. We therefore suggest, based on our findings, that continuous studies are required to enhance the understanding, diagnosis, and treatment of intrahepatic bile duct cancer.

This study is based on secondary data, and to estimate biliary tract cancer patients as accurately as possible from the NHID data, we assessed the initial date of diagnosis for all patients diagnosed with biliary tract cancer as a main disease, between 2002 and 2015. Patients who had a history of biliary tract cancer were excluded from the group of new patients. However, the patients with multiple cancers-due to the lack of knowledge on the primary site, histological diagnosis, and diagnosis methodwere appropriately organized using date of diagnosis, admission or outpatient data, and data on medical expenses, Therefore, diagnostic information on patients with multiple cancers may be less accurate. However, due to legal issues, it was impossible for the investigators to assess the combined data of the NHID and National Cancer Incidence Data. To assess the validity of the incidence rates estimated by the NHID data, we compared our data with the incidence rates from the Korea National Cancer Incidence Database. The incidence rates between the two databases were mostly similar, despite small differences in different years, and the location of the lesion. ${ }^{27}$ This is the second study to assess epidemiological data on the incidence rate of biliary tract cancers in the Korean population since the first study in $2010{ }^{12}$ and is the first to assess the changes in incidence rates for a 10-year period, based on the location of the lesion, and the region within the country.

In conclusion, despite the decreased incidence rate of biliary tract cancers in the Korean population in the past 10 years, the incidence rate is still higher than that of other countries. The 5-year survival rate of biliary tract cancers was also poorer compared to 5-year survival rates for major cancers in South Korea. However, patients who were diagnosed at a relatively younger age exhibited improved 5-year survival. The incidence rates of biliary tract cancers were different between the regions, and the Gyeongnam province region has higher risk for biliary tract cancers compared to other regions. Future studies are 
needed to validate the reason for the decreased incidence rate of biliary tract cancers in Korea. Furthermore, intrahepatic bile duct cancers exhibited a high incidence rate and poor survival rate, and therefore additional studies are needed to further develop cancer prevention and early diagnosis strategies.

\section{CONFLICTS OF INTEREST}

No potential conflict of interest relevant to this article was reported.

\section{ACKNOWLEDGEMENTS}

This work was supported by a grant from National Cancer Center (NCC 1710141-2).

\section{REFERENCES}

1. Chan E, Berlin J. Biliary tract cancers: understudied and poorly understood. J Clin Oncol 2015;33:1845-1848.

2. Jung KW, Won YJ, Oh CM, et al. Cancer Statistics in Korea: incidence, mortality, survival, and prevalence in 2014. Cancer Res Treat 2017;49:292-305.

3. Shaib YH, Davila JA, McGlynn K, El-Serag HB. Rising incidence of intrahepatic cholangiocarcinoma in the United States: a true increase? J Hepatol 2004;40:472-477.

4. Jepsen P, Vilstrup H, Tarone RE, Friis S, Sørensen HT. Incidence rates of intra- and extrahepatic cholangiocarcinomas in Denmark from 1978 through 2002. J Natl Cancer Inst 2007;99:895-897.

5. Utada M, Ohno Y, Tamaki T, Sobue T, Endo G. Long-term trends in incidence and mortality of intrahepatic and extrahepatic bile duct cancer in Japan. J Epidemiol 2014;24:193-199.

6. Flemming JA, Zhang-Salomons J, Nanji S, Booth CM. Increased incidence but improved median overall survival for biliary tract cancers diagnosed in Ontario from 1994 through 2012: a population-based study. Cancer 2016;122:2534-2543.

7. Pinter M, Hucke F, Zielonke N, et al. Incidence and mortality trends for biliary tract cancers in Austria. Liver Int 2014;34:11021108.

8. Khan SA, Taylor-Robinson SD, Toledano MB, Beck A, Elliott P, Thomas HC. Changing international trends in mortality rates for liver, biliary and pancreatic tumours. J Hepatol 2002;37:806-813.

9. West J, Wood H, Logan RF, Quinn M, Aithal GP. Trends in the incidence of primary liver and biliary tract cancers in England and Wales 1971-2001. Br J Cancer 2006;94:1751-1758.

10. Surveillance Research Program, National Cancer Institute. Fast Stats: an interactive tool for access to SEER cancer statistics [Internet]. Bethesda: National Institutes of Health [cited 2018 Jan 10]. Available from: https://seer.cancer.gov/faststats.

11. Parkin DM, Ohshima H, Srivatanakul P, Vatanasapt V. Cholangiocarcinoma: epidemiology, mechanisms of carcinogenesis and prevention. Cancer Epidemiol Biomarkers Prev 1993;2:537-544.
12. Shin HR, Oh JK, Lim MK, et al. Descriptive epidemiology of cholangiocarcinoma and clonorchiasis in Korea. J Korean Med Sci 2010;25:1011-1016.

13. Kim H, Shin AR, Chung HH, et al. Recent trends in hepatitis B virus infection in the general Korean population. Korean J Intern Med 2013;28:413-419.

14. Potkonjak M, Miura JT, Turaga KK, et al. Intrahepatic cholangiocarcinoma and gallbladder cancer: distinguishing molecular profiles to guide potential therapy. HPB (Oxford) 2015;17:1119-1123.

15. Rustagi T, Dasanu CA. Risk factors for gallbladder cancer and cholangiocarcinoma: similarities, differences and updates. J Gastrointest Cancer 2012;43:137-147.

16. Ishihara S, Horiguchi A, Miyakawa S, Endo I, Miyazaki M, Takada T. Biliary tract cancer registry in Japan from 2008 to 2013. J Hepatobiliary Pancreat Sci 2016;23:149-157.

17. Seong SC, Kim YY, Khang YH, et al. Data resource profile: the national health information database of the National Health Insurance Service in South Korea. Int J Epidemiol 2017;46:799-800.

18. Oh CM, Won YJ, Cho H, et al. Alanine aminotransferase and gamma-glutamyl transferase have different dose-response relationships with risk of mortality by age. Liver Int 2016;36:126-135.

19. Korea Central Cancer Registry. Annual report of cancer statistics in Korea in 2014 [Internet]. Goyang: National Cancer Center [cited 2018 Apr 3]. Available from: http://ncc.re.kr/.

20. American Cancer Society. Cancer in children: what are the differences between cancers in adults and children? [Internet]. Atlanta: American Cancer Society; c2017 [cited 2018 Jul 6]. Available from: https://www.cancer.org/cancer/cancer-in-children/differences-adults-children.html.

21. Datta RV, LaQuaglia MP, Paty PB. Genetic and phenotypic correlates of colorectal cancer in young patients. N Engl J Med 2000;342:137-138.

22. Korea Statistical Information Service. Population statistics based on resident registration [Internet]. Daejeon: Statistics Korea [cited 2017 Dec 10]. Available from: http://kosis.kr.

23. Mosadeghi S, Liu B, Bhuket T, Wong RJ. Sex-specific and race/ ethnicity-specific disparities in cholangiocarcinoma incidence and prevalence in the USA: an updated analysis of the 2000-2011 Surveillance, Epidemiology and End Results registry. Hepatol Res 2016;46:669-677.

24. Lepage C, Cottet V, Chauvenet M, et al. Trends in the incidence and management of biliary tract cancer: a French populationbased study. J Hepatol 2011;54:306-310.

25. Hundal R, Shaffer EA. Gallbladder cancer: epidemiology and outcome. Clin Epidemiol 2014;6:99-109.

26. Randi G, Malvezzi M, Levi F, et al. Epidemiology of biliary tract cancers: an update. Ann Oncol 2009;20:146-159.

27. Jeong YI, Shin HE, Lee SE, et al. Prevalence of Clonorchis sinensis infection among residents along 5 major rivers in the Republic of Korea. Korean J Parasitol 2016;54:215-219.

28. Lee BS, Cho YK, Jeong SH, et al. Nationwide seroepidemiology of hepatitis B virus infection in South Korea in 2009 emphasizes the 
coexistence of HBsAg and anti-HBs. J Med Virol 2013;85:13271333.

29. Lee H, Lee H, Cho Y, Oh K, Ki M. Changes in seroprevalence of hepatitis B surface antigen and epidemiologic characteristics in the Republic of Korea, 1998-2013. Epidemiol Health 2015;37:e2015055.

30. Kwon GY, Lee H, Gwack J, Lee SW, Ki M, Youn SK. Regional distribution of hepatitis C virus infection in the Republic of Korea,
2007-2011. Gut Liver 2014;8:428-432.

31. Jeong SH, Jang ES, Choi HY, Kim KA, Chung W, Ki M. Current status of hepatitis $\mathrm{C}$ virus infection and countermeasures in South Korea. Epidemiol Health 2017;39:e2017017.

32. Shon HS, Choi HY, Kim JR, et al. Comparison and analysis of the prevalence of hepatitis $\mathrm{C}$ virus infection by region in the Republic of Korea during 2005-2012. Clin Mol Hepatol 2015;21:249-256. 